\author{
BASILIS C. XANTHOPOULOS \\ Department of Physics, University of Crete, \\ Iraklion, Crete, Greece.
}

Abstract. The local black holes describe physical situations involving a black hole surrounded by a finite vacuum region and then by matter and fields. The stationary and axisymmetric local black holes belong into two classes, the spherical and the toroidal ones, depending on the topology of their horizon. For the static black holes their metric tensors are given explicitly in terms of Legendre polynomials. For the stationary local black holes the problem is formulated interms of the Ernst potential of the rotational killing field and the appropriate asymptotic conditions on the horizon are determined.

\title{
1. LOCAL BLACK HOLES
}

A black hole, being the final configuration of a burnt out and settled down massive star, is described in General Relativity by a stationary spacetime. A stationary isolated black hole - i.e., a spacetime representing a single black hole - has to be axisymmetric ${ }^{1}$ as well and its horizon must have the topology of the sphere $\mathrm{s}^{2}$. In fact the two parametric family of the Kerr ${ }^{2}$ solutions of the Einstein equations describes the most general ${ }^{3-5}$ stationary isolated black hole (We shall not be concerned in this paper with electrically charged black holes). To study in General Relativity physical phenomena involving isolated black holes, we have to use approximation methods. For instance, in all studies $6-7$ of the fall of particles into, or the scattering of waves by, a black hole we treat the falling matter or the scattered field as a perturbation, i,e., we treat them as test Darticles or test fields which move in, or are propagated in, the spacetime of the black hole, without actually affecting the geometry itself.

The concept of the local black holes ${ }^{8}$ represents the first step in an effort to describe exactly in General Relativity physical phenomena involving a black hole surrounded by matter and fields. Since it is reasonable to assume that the matter that was in the immediate neighborhood of the black hole has been "eaten up" by the hole, we demand that the black hole is surrounded, for a finite distance from its horizon, by a vacuum region. No matter or any other field but the gravitational field exists in this vacuum region. However, matter and other fields may exist (and generally do exist) farther away from this vacuum region; it is only de425

\section{G. O. Abell and G. Chincarini (eds.), Early Evolution of the Universe and Its Present Structure, 425-430. (C) 1983 by the IAU.}


manded that their density and strength fall off sufficiently fast away from the hole so that they represent an isolated system with a black hole at the center.

The general theory of the static and axisymmetric local black holes has been recently put forward by Geroch and Hartle ${ }^{8}$. In particular they have shown that the horizon of these black holes is homeomorphic either to the sphere $S^{2}$ or the torus $S^{1} \mathrm{xS}^{1}$; these two classes will be referred to as the spherical and the toroidal black holes, respectively. Besides indicating how to construct such solutions, Geroch and Hartle have investigated their global structure, their thermodynamic behavior and their evolution with the emission of Hawking radiation. Certain examples of local black holes had appeared in the literature ${ }^{9}-11$ before the work of Geroch and Hartle.

\section{THE STATIC AND AXISYMMETRIC LOCAL BLACK HOLES}

The construction of the spacetime of the local black holes proceeds in two steps ${ }^{8}$. In the first we are concerned with the immediate (the vacuum) neighborhood of the black hole, where the actual difficulty is to satisfy the vacuum Einstein, equations compatibly with the requirement that they admit a smooth event horizon. The second step then will be to match this solution (of the Einstein equations) with an exterior solution with sources, which will represent the region far away from the hole. All the studies of the local black holes are concerned, so far, with the first step. For the static and axisymmetric case in particular the metric tensors which describe the vacuum neighborhoods of the spherical and the toroidal black holes have been obtained explicitly by Chandrasekhar 7 and Xanthopoulos ${ }^{12}$ respectively. Here we give only the results.

Spherical black holes ${ }^{7}$ : The line element is

$$
\begin{aligned}
& d s^{2}=\frac{(n-1)}{(n+1)} e^{S}(d t)^{2}-\frac{m^{2}(n+1)}{n-1} e^{\sigma-S}(d n)^{2}- \\
& -m^{2}(n+1)^{2} e^{-S}\left\{\left(1-\mu^{2}\right)(d \varphi)^{2}+\left(1-\mu^{2}\right)^{-1} e^{\sigma}(d \mu)^{2}\right\},
\end{aligned}
$$

where $S=S(n, \mu)=\sum A_{k} P_{k}(n) P_{k}(\mu)$ and the function $\sigma=\sigma(n, \mu)$ is determined from $S$ by the equations

$$
\begin{aligned}
& \frac{\left(n^{2}-\mu^{2}\right)}{\left(n^{2}-1\right)\left(1-\mu^{2}\right)} \sigma, \eta=\frac{2 n}{n^{2}-1} S, \eta^{-}-\frac{2 \mu}{n^{2}-1} S, \mu-\mu S,{ }_{n}{ }^{2}, \mu+ \\
& +\frac{n}{2\left(n^{2}-1\right)}\left\{\left(n^{2}-1\right) s_{,}^{2}+\left(\mu^{2}-1\right) s, \underset{\mu}{2}\right\} \\
& \frac{\left(n^{2}-\mu^{2}\right)}{\left(n^{2}-1\right)\left(1-\mu^{2}\right)} \sigma, \mu=\frac{2 \mu}{1-\mu^{2}} S_{, n}+\frac{2 n}{n^{2}-1} S_{, \mu}+n S_{, n} S_{, \mu}+ \\
& +\frac{\mu}{2\left(1-\mu^{2}\right)}\left\{\left(n^{2}-1\right) S, \underset{n}{2}+\left(\mu^{2}-1\right) S, \underset{\mu}{2}\right\}
\end{aligned}
$$


The $P_{k}$ 's are Legendre polynomials and the constants $A_{k}$ are subject to the condition $\sum A_{2 k+1}=0$. The mass of the hole is $m$, its horizon is the surface $n=1$, the surface area of the horizon is $16 \pi \mathrm{m}^{2} \mathrm{e}^{\alpha}$ and the surface gravity on the horizon is $\left(4 \pi e^{\alpha}\right)^{-1}$, where $\alpha=-\sum A_{2 k}$. (All the summations are from zero to infinity).

Toroidal black holes.12: The line element is

$$
\begin{aligned}
& d s^{2}=-\frac{1}{4}\left(n^{2}-1\right)\left(1-\mu^{2}\right) e^{-S}(d t)^{2}-4 m^{2} e^{S}(d \varphi)^{2}- \\
& -4 m^{2}\left(n^{2}-\mu^{2}\right) e^{\sigma-S}\left\{\left(n^{2}-1\right)^{-1}(d n)^{2}+\left(1-\mu^{2}\right)^{-1}(d \mu)^{2}\right\},
\end{aligned}
$$

where $S=S(n, \mu)=\sum B_{k} P_{k}(n) P_{k}(\mu)$ and the function $\sigma=\sigma(n, \mu)$ is determined from $S$ by the equations

$$
\begin{aligned}
& \sigma, \eta=-\frac{n\left(\mu^{2}-1\right)}{2\left(n^{2}-\mu^{2}\right)}\left\{\left(\eta^{2}-1\right) S,{ }_{n}^{2}+\left(\mu^{2}-1\right) S,{ }_{\mu}^{2}\right\}+\frac{\mu\left(\eta^{2}-1\right)\left(\mu^{2}-1\right)}{n^{2}-\mu^{2}} S,{ }_{n} S, \mu \\
& \sigma,{ }_{\mu}=\frac{\mu\left(n^{2}-1\right)}{2\left(n^{2}-\mu^{2}\right)}\left\{\left(n^{2}-1\right) S,{ }_{n}^{2}+\left(\mu^{2}-1\right) S,{ }_{\mu}^{2}\right\}-\frac{n\left(n^{2}-1\right)\left(\mu^{2}-1\right)}{n^{2}-\mu^{2}} S,{ }_{n} S, \mu
\end{aligned}
$$

The $P_{k}$ 's are Legendre polynomials and the constants $B_{k}$ are subject to the conditions $\sum \mathrm{B}_{2 k+1}=0, \sum \mathrm{B}_{2 k} \dot{\mathrm{P}}_{2 k}(1)=0$, and $\sum \mathrm{B}_{2 k+1} \ddot{\mathrm{P}}_{2 k+1}(1)=0$, where the dots denote differentiations. The mass of the hole is $m$, the surface area of the horizon is $16 \mathrm{~mm}^{2} \mathrm{e}^{\alpha}$ and the surface gravity on the horizon is $\left(4 m e^{\alpha}\right)^{-1}$, where $2 \alpha$ equals to the constant value of the function $\sigma$ on the horizon.

Geroch and Hartle ${ }^{8}$ have shown that the above solutions can be smoothly continued to asymptotically flat non-vacuum solutions. It should be mentioned, however, that no non-vacuum continuation has been explicitly constructed so far corresponding to some physically interesting distribution of matter.

\section{THE STATIONARY CASE}

Contrary to the static black holes, which are non-rotating, the stationary and axisymmetric black holes are uniformly rotating. Unfortunately, the relevant stationary axisymmetric vacuum Einstein equations are quite complicated. For instance, in the static case a combination of the components of the metric tensor satisfies a single linear equation; in the stationary case, instead, we have to deal with a non-linear system of partial differential equations. In particular we choose a coordinate chart which covers a neighborhood of the horizon and we determine the asymptotic behaviors of the coefficients of the metric tensor which guarantee the existence of a smooth horizon. We find that a formulation of the problem based on the Ennst potential ${ }^{3}$ associated with the rotational Killing field is the most appropriate. Finally, we determine a simple necessary condition which distinguishes the spherical and the toroidal holes.

A suitable expression for the line element of the general stationary 
axisymmetric spacetime is

$d s^{2}=t e^{2 \nu}(d t)^{2}-e^{2 \psi}(d \varphi-\omega d t)^{2}-e^{2 \mu 2}\left(d x^{2}\right)^{2}-e^{2 \mu} 3\left(d x^{3}\right)^{2}$,

where $t$ and $\varphi$ are the time and the azimuthal angle and the scalars $\nu, \psi, \omega, \mu_{2}$ and $\mu_{3}$ are functions of the two remaining spatial coordinates $\mathrm{x}^{2}$ and $\mathrm{x}^{3}$. The Einstein equations for the stationary and axisymmetric case can be found, for instance, in reference 7 equations $7,8,14,15$, and 16 of chapter VI. We note that eq. 14, compatibly with which the gauge condition should be imposed ${ }^{14}$, is the same in both the static and the stationary cases. We can choose the gauge, therefore, exactly as in the static case. Since the argument is presented in detail in ref. 12, we here give only the conclusions: We impose the gauge conditions

$$
e^{\mu_{3}-\mu_{2}}=\left(n^{2}-1\right)^{1 / 2}\left(1-\mu^{2}\right)^{-1 / 2}, \quad e^{\psi+\nu}=m\left(n^{2}-1\right)^{1 / 2}\left(1-\mu^{2}\right)^{1 / 2}
$$

for which the line element (7) becomes

$$
\begin{aligned}
& d s^{2}=m\left(n^{2}-1\right)^{1 / 2}\left(1-\mu^{2}\right)^{1 / 2}\left\{e^{\nu-\psi}(d t)^{2}-e^{\psi-\nu}(d \varphi-\omega d t)^{2}\right\}- \\
& -\left(n^{2}-1\right)^{1 / 2}\left(1-\mu^{2}\right)^{1 / 2} e^{\mu} 2^{+\mu} 3\left\{\left(n^{2}-1\right)^{-1}(d n)^{2}+\left(1-\mu^{2}\right)^{-1}(d \mu)^{2}\right\} .
\end{aligned}
$$

Here $m$ is a constant, the range of $\mu=x^{3}$ is in $(-1,+1)$, and the horizon is the surface $n=x^{2}=1$.

The limit on the horizon of the determinant of the spacetime metric is

$$
G_{(4)}=-2 m^{2}\left(1-\mu^{2}\right) \lim \left\{(n-1) e^{2\left(\mu_{2}+\mu_{3}\right)}\right\}
$$

and the limit on the horizon of the corresponding determinant of the induced metric is

$$
G_{(2)}=2 m \lim \left\{(n-1) e^{\psi-\nu} e^{\mu_{2}+\mu_{3}}\right\} \text {. }
$$

For the metric (9) to be well behaved on the horizon the limits $G_{(4)}$ and $G_{(2)}$ should be finite and non-zero. Therefore, we should also have that

$$
\lim \left\{(n-1)^{1 / 2} e^{\psi-\nu}\right\} \text { and } \lim \left\{(n-1)^{1 / 2} e^{\mu_{2}+\mu_{3}}\right\}
$$

are finite and non-zero. We observe that we have obtained the same asymptotic conditions as in the static case 12 .

By glancing on themetric (9) one can immediately see that the first of the conditions (12) is equivalent to the condition that the squared norm of the rotational Killing field is finite and non-zero on the horizon. This observation suggests to use the formulation of the stationary axisymmetric Einstein equations interms of the Ernst potential13-14 associated with the rotational Killing field. Hence, instead of the variables $\psi-\nu$ and $\omega$ we consider

$$
\Psi=\left(n^{2}-1\right)^{1 / 2}\left(1-\mu^{2}\right)^{1 / 2} e^{\psi-\nu}
$$

and $\Phi$ defined by the equations

$$
\left(n^{2}-1\right) \Phi, \eta=\Psi^{2} \omega, \mu \quad \text { and } \quad\left(\mu^{2}-1\right) \Phi, \mu=\Psi^{2} \omega, \eta,
$$


as the basic variables of the problem. In terms of the complex Ernst potential $Z=\Psi+i \Phi$ the vacuum Einstein equations read

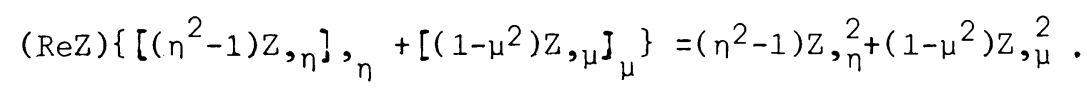

In addition, instead of $\mu_{2}+\mu_{3}$, we consider the $M$ defined by

$$
e^{\mu_{2}+\mu_{3}}=\left(n^{2}-\mu^{2}\right)\left(n^{2}-1\right)^{1 / 2}\left(1-\mu^{2}\right)^{-1 / 2} \psi^{-1} e^{M}
$$

as an equivalent variable. Interms of these variables the line element (9) takes the form

$$
\begin{aligned}
& d s^{2}=m\left\{\left(n^{2}-1\right)\left(1-\mu^{2}\right) \Psi^{-1}(d t)^{2}-\Psi(d \varphi-\omega d t)^{2}\right\}- \\
& -\left(n^{2}-\mu^{2}\right) \Psi^{-1} e^{M}\left\{\left(n^{2}-1\right)^{-1}(d n)^{2}+\left(1-\mu^{2}\right)^{-1}(d \mu)^{2}\right\} .
\end{aligned}
$$

Note that, the second of the asymptotic conditions (12) is that $e^{M}$ should be finite and non-zero on the horizon.

Turning now to the remaining of the Einstein equations-namely, equations 8 and 16 of chapter VI of the reference 7- we obtain, after a lengthy calculation, that $M$ is determined from $\Psi$ and $\Phi$ by the equations

$$
\begin{aligned}
& 2 M,_{n}=\left(1-\mu^{2}\right)\left(n^{2}-\mu^{2}\right)^{-1}\left\{n A-2 \mu\left(n^{2}-1\right) B\right\} \\
& 2 M,_{\mu}=\left(n^{2}-1\right)\left(n^{2}-\mu^{2}\right)^{-1}\left\{\mu A+2 n\left(1-\mu^{2}\right) B\right\},
\end{aligned}
$$

where

$$
\begin{aligned}
& \mathrm{A}=\Psi^{-2}\left\{\left(\eta^{2}-1\right)\left(\Psi,{ }_{n}^{2}+\Phi,{ }_{\eta}^{2}\right)+\left(\mu^{2}-1\right)\left(\Psi,,_{\mu}^{2}+\Phi,,_{\mu}^{2}\right)\right\} \\
& \mathrm{B}=\Psi^{-2}\left(\Psi,{ }_{\eta} \Psi,{ }_{\mu}+\Phi,{ }_{\eta} \Phi,{ }_{\mu}\right) .
\end{aligned}
$$

Note that by using equation (15) and the assumption that $\lim \Psi$ is finite and nonczero we can conclude that $\Phi$ is smooth in a neighborhood of the horizon. Hence $A$ and $B$ are bounded in a neighborhood of the horizon and the second of equations (18) implies that $M$ is constant on the horizon. Therefore the second of the asymptotic conditions (12) is satisfied as a consequence of the first asymptotic condition.

Finally we obtain an additional necessary condition on the scalar $\Psi$ by applying the Gauss-Bonnet ${ }^{15}$ theorem. The induced on the horizon metric from the spacetime metric is

$$
d \tau^{2}=m g(d \varphi)^{2}+g^{-1} e^{M}(d \mu)^{2}
$$

where $g=g(\mu)=\Psi(1, \mu)$ and $M$ now stands for the constant value of the function $M(n, \mu)$ on the horizon. The scalar curvature of the metric (20) is easily found to be $R=-\ddot{g} e^{-M}$, where the dots denote differentiations. Then the Gauss-Bonnet formula $4 \pi x=\int R d V$ gives that

$$
2 x \mathrm{~m}^{-1 / 2} e^{\mathrm{M} / 2}=\dot{\mathrm{g}}(-1)-\dot{\mathrm{g}}(+1) \text {. }
$$

In the expression (21) $x$ is the Euler number ${ }^{16}$ of the horizon of the black hole, which is a topological invariant; $x=2$ for a spherical and $x=0$ for a toroidal horizon. We conclude therefore that $g$ has to satisfy the con- 
dition

$$
\dot{g}(-1)-\dot{g}(+1)=\left[\begin{array}{cc}
4 m^{-1 / 2} e^{M / 2} & \text { for a spherical black hole. } \\
0 & \text { for a toroidal black hole. }
\end{array}\right.
$$

We summarize the conclusions of this section: The stationary and axisymmetric local black holes should be searched among the smooth solutions of the Ernst equation (15) whose real part $\Psi$ of the complex potential $Z$ is finite and different from zero on the horizon $n=1$ and it satisfies, in addition, the condition (22). The metric tensor is given by equation (17) where $\omega$ and $M$ are determined from $\Psi$ and $\Phi$ via the equations (14) and (18).

\section{REFERENCES}

1. Hawking, S.W.: 1972, Commun. Math. Phys. 25, pp. 152-166.

2. Kerr, R.P.: 1963, Phys. Rev. Lett. 11, pp. 237-8.

3. Israel, W.: 1968, Phys. Rev. 164, pp. 1776-9.

4. Carter, B.: 1972, Phys. Rev. Lett. 26, pp. 331-3.

5. Robinson, D.C.: 1975, Phys. Rev. Lett. 34, pp. 905-6.

6. Misner, C.W., Thorne, K.S, and Wheeler, J.A.: 1973, "Gravitation", W.H. Freeman and Company, San Francisco.

7. Chandrasekhar, S.: 1982, "The mathematical theory of black holes", Oxford at the Clarendon Press.

8. Geroch, R., and Hartle, J.B.: 1982, J. Math. Phys. 23, pp. 680-92.

9. Israel, W., and Khan, K.A.: 1964, Nuovo Cim., 33, pp. 331-44.

10. Mysak, L.A,, and Szekeres, G.: 1966, Can. J. Phys. 44, pp. 617-

11. Peters, P.C.: 1979, J. Math. Phys. 20, pp. 1481-5.

12. Xanthopoulos, B.C.; 1982, "Local toroidal black holes that are static and axisymmetric", Proc. R. Soc. Lond. (submitted).

13. Ernst, F.J.: 19.68, Phys. Rev. 167, pp. 1175-8.

14. Chandrasekhar, S.: 1978, Proc. R. Soc. Lond. A358, pp. 405-20.

15. Hicks, N, J.: 1971,"Notes on differential geometry", Van Nostrand Reinhold Company, London.

16. Steenrod, N.: 1951, "The topology of fibre bundles", Princeton University Press.

\section{Discussion}

Novikov: Is the toroidal black hole stable against the small perturbations? I guess it may be unstable.

Xanthopoulos: It is unknown. 